\title{
Hydrogen-induced plasticity in nanoporous palladium
}

\author{
Markus Gößler ${ }^{* 1}$, Eva-Maria Steyskal ${ }^{1}$, Markus Stütz ${ }^{2}$, Norbert Enzinger ${ }^{2}$ \\ and Roland Würschum ${ }^{1}$
}

\author{
Full Research Paper \\ Address: \\ ${ }^{1}$ Institute of Materials Physics, Graz University of Technology, \\ Petersgasse 16, A-8010 Graz, Austria and ${ }^{2}$ Institute of Materials \\ Science, Joining and Forming, Graz University of Technology, \\ Kopernikusgasse 24/I, A-8010 Graz, Austria \\ Email: \\ Markus Gößler ${ }^{*}$ - m.goessler@tugraz.at; Roland Würschum - \\ wuerschum@tugraz.at \\ * Corresponding author \\ Keywords: \\ electrochemistry; hydride formation; in situ dilatometry; internal-stress \\ plasticity; nanoporous palladium
}

Beilstein J. Nanotechnol. 2018, 9, 3013-3024.

doi:10.3762/bjnano.9.280

Received: 29 June 2018

Accepted: 11 November 2018

Published: 10 December 2018

Associate Editor: P. Leiderer

(C) 2018 Gößler et al.; licensee Beilstein-Institut.

License and terms: see end of document.

\begin{abstract}
The mechanical strain response of nanoporous palladium (npPd) upon electrochemical hydrogenation using an in situ dilatometric technique is investigated. NpPd with an average ligament diameter of approximately $20 \mathrm{~nm}$ is produced via electrochemical dealloying. A hydrogen-induced phase transition from $\mathrm{PdH}_{\beta}$ to $\mathrm{PdH}_{\alpha}$ is found to enable internal-stress plasticity (or transformationmismatch plasticity) in nanoporous palladium, which leads to exceptionally high strains without fracture as a result of external forces. The high surface stress in the nanoporous structure in combination with the internal-stress plasticity mechanism leads to a peculiar strain response upon hydrogen sorption and desorption. Critical potentials for the formation of $\mathrm{PdH}_{\alpha}$ and $\mathrm{PdH}_{\beta}$ in $n p P d$ are determined. The theoretical concepts to assess the plastic strain response of nanoporous samples are elucidated, taking into account characteristics of structure and deformation mechanism.
\end{abstract}

\section{Introduction}

Material properties on the nanoscale can differ substantially from their bulk counterparts considering the increasing importance of surface effects. The high surface-to-volume ratios in such materials allow for the control of bulk features by surface modifications. Electrostatic charging or electrochemical (surface) reactions are possible ways to influence metal surfaces in a well-defined manner. Therefore, open nanoporous network structures are particularly suitable for property-tuning experiments in an electrochemical environment, due to a large contact area with the electrolyte and macroscopic sample dimensions.
In nanoporous metals, the electrochemical control of actuation [1-3], resistance [4-6], magnetic moment [5,7], optical transmission [8] and selective chemical transport [9] have been reported in recent years, apart from the mechanical properties described below.

Dealloying, a selective dissolution process, has become an established technique to produce metallic nanoporous structures. By exposing a (binary) alloy to an etching agent, the less noble component is gradually removed, while enhancing the 
surface diffusivity of the other. Surface diffusion is of special importance for the formation of nanoporous networks, as passivation of the alloy surface due to a flawless coverage by the noble component prevents the etching process. After Erlebacher et al. delivered a full description of the dealloying process on an atomistic scale [10], the process itself has been extensively studied [11-13], while being used to prepare a broad variety of different nanoporous metals [14,15].

Lately, dealloyed nanoporous palladium (npPd) structures [16] have received attention in the literature as electrochemical actuator materials [17-20]. Due to the ability of palladium to host hydrogen atoms in its crystal lattice, such actuators show exceptionally strong, reversible expansion. This distinguishes palladium from other metals in the same group of the periodic table, such as platinum or nickel, which are only capable of adsorbing hydrogen mainly on their surfaces. In the literature, a solubility of hydrogen in palladium up to concentrations of about 0.7 $(\mathrm{H} / \mathrm{Pd})$ has been reported for loading from the gas phase at a hydrogen pressure of the order of $30 \mathrm{kPa}[21]$ at $20^{\circ} \mathrm{C}$. In this compositional range two different phases coexist: At hydrogen concentrations below $0.02(\mathrm{H} / \mathrm{Pd})$ only the $\alpha$-phase (solid solution, $\mathrm{PdH}_{\alpha}$ ) is observed, while at higher concentrations the $\beta$-phase (hydride, $\mathrm{PdH}_{\beta}$ ) starts to form. At concentrations above $0.6(\mathrm{H} / \mathrm{Pd})$ only $\mathrm{PdH}_{\beta}$ is present. These critical concentrations were reported for bulk palladium samples at room temperature and may vary for nanoscaled or stressed systems [22]. The lattice constant increases during hydrogen sorption from $3.887 \AA$ for pure palladium to $3.895 \AA$ for $\mathrm{PdH}_{\alpha}$ (maximum hydrogen concentration at $25^{\circ} \mathrm{C}$ ) and amounts to $4.025 \AA$ in $\mathrm{PdH}_{\beta}$ (minimum hydrogen concentration at $25^{\circ} \mathrm{C}$ ) [21]. This significant increase in lattice constants, and thus volumetric expansion upon hydrogen uptake, makes palladium also an interesting element for hydrogen-sensing applications [23].

The mechanical properties of nanoporous samples have been extensively studied in the literature, especially for the model system of nanoporous gold (npAu). Reports on potential-controlled creep [24], fracture [25] and strength [26] in npAu add to the list of above-mentioned tunable properties in nanoporous metals. The deformation mechanism in such structures has been discussed in detail. Since nanoporous materials exhibit high surface-to-volume ratios, moving dislocations may escape crystals via the surface, which may lead to a scenario of dislocation starvation [27]. This dislocation starvation also implies that the work hardening mechanism, which is based on dislocation interactions, is not active. However, current literature suggests that the active deformation mechanism in $\mathrm{npAu}$ is dislocation slip [28] and that dislocation starvation is not effective at low strains in npAu. Despite the local dislocation activity in the ligaments, macroscopic plasticity, involving dislocations travel- ling larger distances in the network structure, is hard to achieve in nanoporous metals [15]. Plastic deformation in npPd has not been the subject of experimental studies up to this point. This work focuses on the strain response of npPd upon hydrogenation and aims to shed light on the active deformation mechanisms.

\section{Results}

\section{Electrochemical characterisation}

A typical strain response of npPd was measured using an in situ dilatometer setup during a cyclic voltammogram $(\mathrm{CV})$ in $1 \mathrm{M} \mathrm{KOH}$ (see Figure 1). A CV probes the current as a function of the potential, which is varied up and down triangularly at a certain scan rate, which represents the slope of this potential variation.

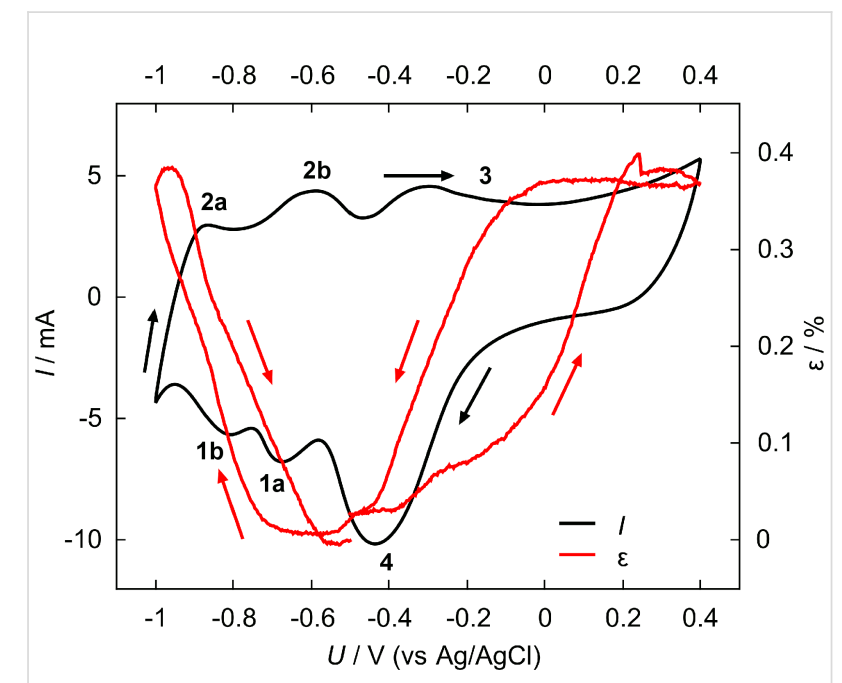

Figure 1: Current I (black) and corresponding strain $\varepsilon$ (red) as functions of the applied potential $U$ during a single voltammetric cycle for nanoporous palladium recorded at a scan rate of $0.5 \mathrm{mV} \cdot \mathrm{s}^{-1}$ in $1 \mathrm{M} \mathrm{KOH}$, zero on the strain axis was chosen in the double-layer regime, arrows indicate the cycling direction of the curves, numbered peaks are discussed in the text.

\section{Potentiostatic hydrogen sorption}

The CV (black) in Figure 1 is typical for palladium and has been discussed in detail in prior studies [18]. Summarising briefly, the negative current peaks below $-0.6 \mathrm{~V}$ (peaks $1 \mathrm{a}$ and 1b) correspond to hydrogen ad- and absorption, while the positive current peaks (peaks $2 \mathrm{a}$ and $2 \mathrm{~b}$ ) are attributed to the reverse process of hydrogen desorption. Above $-0.4 \mathrm{~V}$ the broad flank at positive currents (peak 3) and the large peak at negative currents (peak 4) are features attributed to the adsorption and desorption of oxygen species. It should be noted that the comparably broad peaks strongly depend on the scan rate and exhibit a more distinct shape at lower scan rates. The strain signal (red curve in Figure 1) shows a reversible expansion 
upon hydrogen ad- and absorption below $-0.6 \mathrm{~V}$, while a quasireversible expansion, attributed to the formation of a palladiumoxide species on the surface, is observed at potentials higher than $-0.4 \mathrm{~V}$. The term quasi-reversible refers to the small offset accumulated after an oxide half-cycle, which can be attributed to weak anodic palladium (oxide) dissolution [29]. The length changes upon voltammetric cycling are in agreement with recent results for dealloyed nanoporous palladium from different base alloys $[17,18]$. The highly reversible strain response upon hydrogen sorption and desorption in the $\mathrm{CV}$ motivated a more detailed investigation in this potential regime. Figure 2 shows the strain measured during a series of hydrogen sorption (grey background) and desorption (white background) experiments with decreasing polarisation potentials, but a fixed unloading potential $(-0.4 \mathrm{~V})$. The potential for each sorption step shown is given in the plot. At a potential of $-0.8 \mathrm{~V}$ a first significant reversible change in sample length of about $0.2 \%$ is observed, which increases with more negative sorption potentials to a value of about $0.4 \%$ at $-0.95 \mathrm{~V}$. Obviously, the last loading step at a potential of $-1 \mathrm{~V}$ and the corresponding unloading procedure clearly differ in shape and height from the preceding ones.

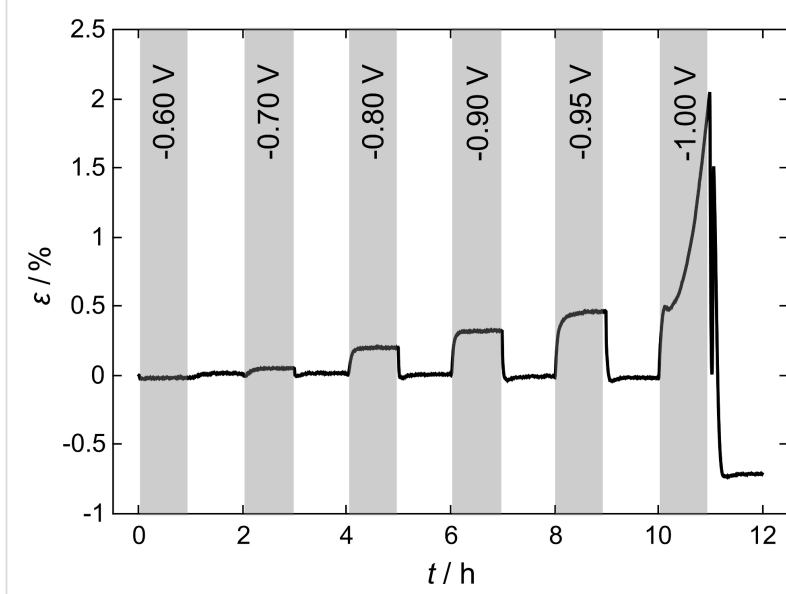

Figure 2: Strain $\varepsilon$ as a function of the time $t$ for a series of consecutive hydrogen absorption/desorption processes at constant potentials in $1 \mathrm{M} \mathrm{KOH}$ for nanoporous palladium. Loading processes are shaded grey, polarisation potentials are given in the grey bars, the unloading potential was fixed at $-0.4 \mathrm{~V}$.

Figure 3 shows a detailed view of this hydrogen loading cycle at a potential of $-1.0 \mathrm{~V}$ and the corresponding unloading procedure at $-0.4 \mathrm{~V}$. Upon hydrogen absorption two different processes can be distinguished in the strain curve: a steep increase up to a strain of about $0.5 \%$, where the curve levels out (region (a)), and a parabolic increase from this point up to the onset of hydrogen desorption (region (b)). The beginning of desorption is marked by a sharp decrease in length (region (c)), followed by a short interval of sharp increase (region (d)), which stands in contrast to a simple picture where a monotonous shrinkage due to hydrogen extraction would be expected. The last stage of hydrogen desorption (region (e)) exhibits a similar slope to region (c) until the strain curve flattens out after complete desorption. The peak in the strain curve separating regions (d) and (e) will be referred to as strain "overshoot" in the following. An irreversible decrease of the sample length of about $0.7 \%$ was detected at the end of the experiment. This irreversible contribution to the total strain could be reproduced in measurements on further samples upon similar treatment.

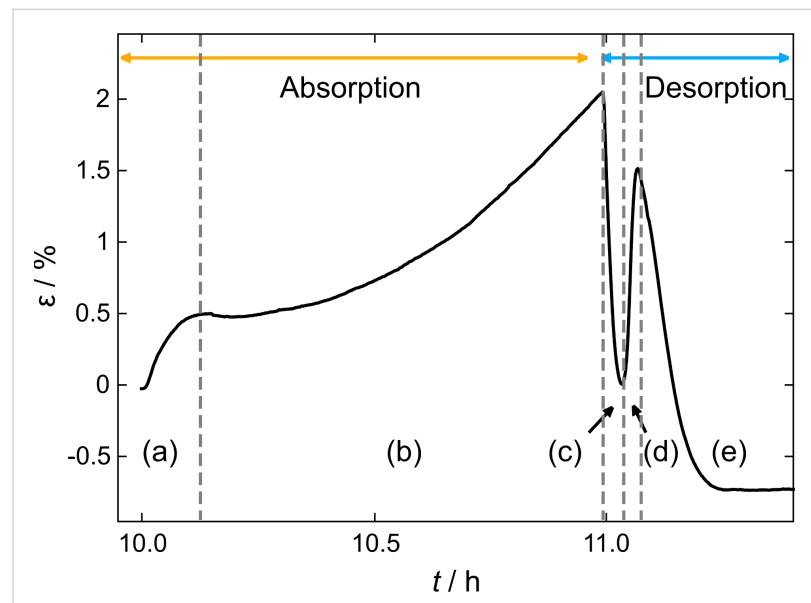

Figure 3: Enlarged view of Figure 2: Strain $\varepsilon$ as a function of time $t$ for electrochemical hydrogen absorption in nanoporous palladium at an anodic potential of $-1.0 \mathrm{~V}$ followed by desorption at $-0.4 \mathrm{~V}$ in $1 \mathrm{M} \mathrm{KOH}$. Labelled regions are discussed in the text (regions (d) and (e): strain overshoot).

\section{Critical potentials of palladium hydride formation}

A series of potentiostatic hydrogen loading and unloading measurements with decreasing loading potentials was carried out ex situ in order to distinguish the phases present in npPd at a certain potential. This procedure is thermodynamically equivalent to the measurement of pressure-composition isotherms (PCIs) upon hydrogen pressurisation. Since the parasitic process of hydrogen evolution contributes to the charge transfer during hydrogen loading, especially at strongly negative potentials, a calculation based on the recorded charge flow during hydrogenation might overestimate the hydrogen concentration in the samples. Therefore, the atomic ratio of hydrogen and palladium $\mathrm{H} / \mathrm{Pd}\left(c_{\mathrm{f}}\right)$ was determined based on the potentiostatic desorption half-cycles. For each desorption step the imposed charge $Q$ was determined by means of trapezoidal integration and converted into $c_{\mathrm{f}}$ considering the sample mass $m$ using the following relation:

$$
c_{\mathrm{f}}=\frac{Q \cdot M_{\mathrm{Pd}}}{e \cdot N_{\mathrm{A}} \cdot m},
$$


where $M_{\mathrm{Pd}}$ is the molar mass of palladium, $M_{\mathrm{Pd}}=106.42 \mathrm{~g} / \mathrm{mol}$, and $e$ and $N_{\mathrm{A}}$ are the elementary charge and the Avogadro constant, respectively. The hydrogen concentration is plotted as a function of the polarisation potential for npPd in Figure 4. Error bars, determined via the uncertainty of trapezoidal integration, are added in the graph, but are small compared to the plot symbols.

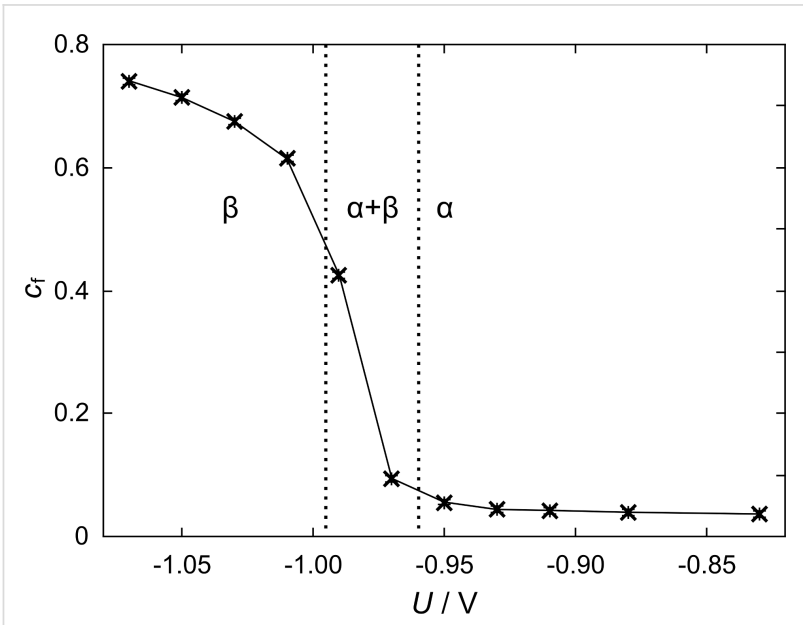

Figure 4: Atomic ratio of hydrogen and palladium $c_{f}(H: P d)$ plotted as function of the polarisation potential $U$ for nanoporous palladium in $1 \mathrm{M} \mathrm{KOH}$, dotted vertical lines separate the regimes of $\mathrm{PdH}_{\alpha}, \mathrm{PdH}_{\beta}$ and phase coexistence.

From the literature it is known that a distinction of palladium hydride phases is possible utilising potential-concentration plots as in Figure 4 [30-32]. In our nanoporous samples the $\mathrm{PdH}_{\beta}$-phase begins to form at potentials below $-0.96 \mathrm{~V}$. Pure
$\mathrm{PdH}_{\alpha}$ is present at potentials higher than $-0.96 \mathrm{~V}$, while $\mathrm{PdH}_{\alpha}$ disappears at potentials below $-0.99 \mathrm{~V}$. Nanostructured materials can exhibit a significant narrowing of the miscibility gap in the phase diagram compared to the bulk metal [33], which can drive the onset of $\beta$-phase formation to higher hydrogen concentrations and/or lower potentials. For nanoporous palladium produced in this work, a $c_{\mathrm{f}}$ value of about 0.07 for the $\beta$-phase onset can be estimated from the data presented in Figure 4, which is in good agreement with a value of 0.06 determined for palladium nanoparticles [30]. The saturation of $\mathrm{PdH}_{\beta}$ formation and the corresponding depletion of $\mathrm{PdH}_{\alpha}$ at a value below $-0.99 \mathrm{~V}$ is consistent with values reported for palladium thin films [31,32]. The threshold values for the palladium hydride $\beta$-phase formation $(-0.96 \mathrm{~V})$ and the potential of $\alpha$-phase depletion $(-0.99 \mathrm{~V})$ can be used for a phase distinction in currentcontrolled experiments.

\section{Galvanostatic hydrogen sorption}

In order to clarify the origin of the unusual strain overshoot observed in Figure 3, a charge-controlled sorption experiment was performed (Figure 5). A constant current of $1 \mathrm{~mA}$ was used to charge nanoporous samples to different total amounts of charge (from $1 \mathrm{~A} \cdot \mathrm{s}$ to $13 \mathrm{~A} \cdot \mathrm{s}$ in total, black curves) and, thus, hydrogen concentrations (compare Equation 1), while for desorption (red curves) the potential was held constant at $-0.4 \mathrm{~V}$ similar to the potentiostatic experiment above (Figure 2). Monitoring the potential during the charging procedure revealed a transgression of the $\mathrm{PdH}_{\beta}$ formation threshold in the fifth loading cycle, which is marked as "onset of $\mathrm{PdH}_{\beta}$ " in Figure 5. The potential of $\mathrm{PdH}_{\alpha}$ depletion was crossed during the 11th loading cycle, which is also indicated in Figure 5.

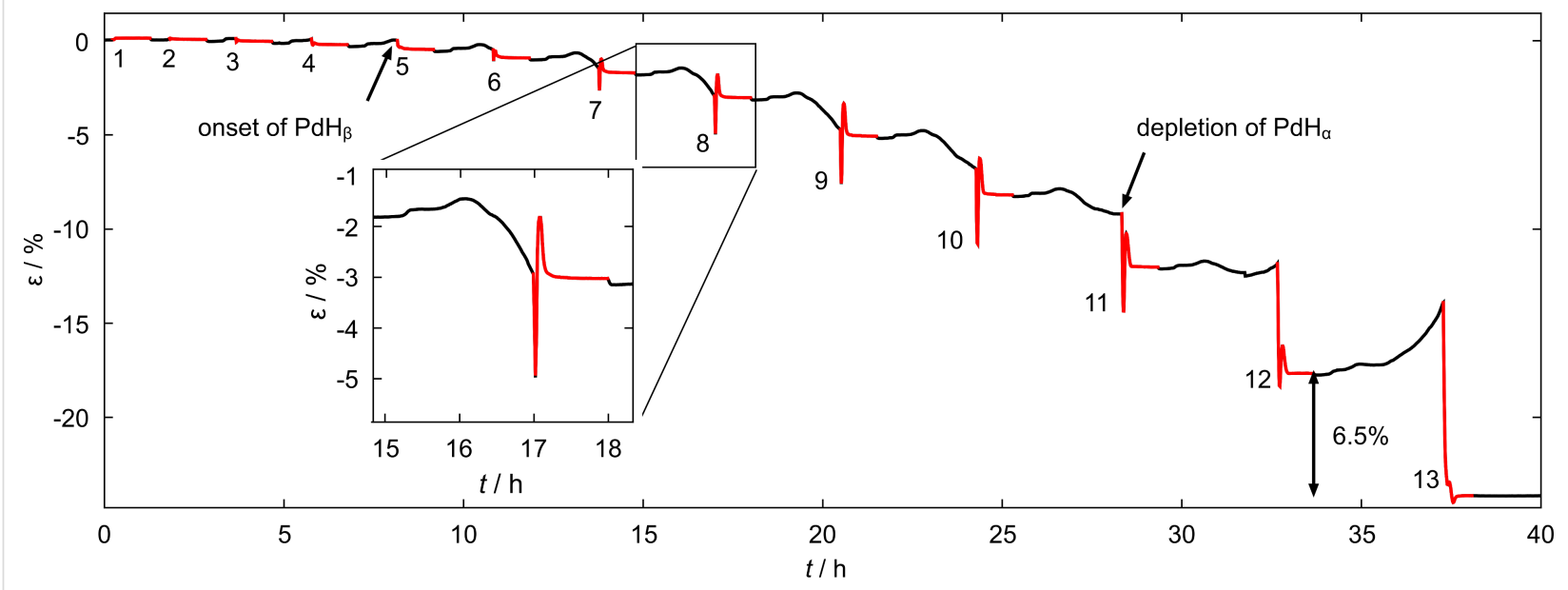

Figure 5: Strain $\varepsilon$ as a function of the time $t$ for a series of consecutive hydrogen absorption processes at a constant current of $1 \mathrm{~mA}$ and desorption processes at $-0.4 \mathrm{~V}$ in $1 \mathrm{M} \mathrm{KOH}$ for nanoporous palladium. Desorption processes are marked red, the amount of charge during hydrogen loading (black) was controlled via the sorption time and increased in steps of $1 \mathrm{~A} \cdot \mathrm{s}$ from $1 \mathrm{~A} \cdot \mathrm{s}$ to $13 \mathrm{~A} \cdot \mathrm{s}$. 
Both strain features present in potentiostatic experiments during desorption, namely the strain overshoot and the irreversible strain offset after a loading/unloading cycle, can be followed in Figure 5 evolving over time. During the first four cycles the strain amplitude upon absorption remains small of the order of $0.2 \%$, with no obvious irreversible contribution. Furthermore, no overshoot can be detected in these initial cycles. In the fifth cycle, the strain upon absorption increases up to $0.4 \%$ and an irreversible strain contribution after desorption of about $-0.3 \%$ emerges. Starting with the sixth cycle the strain overshoot becomes more and more pronounced each step (red curves in Figure 5). Moreover, the strain offset grows drastically up to a value of $6.5 \%$ for the last desorption process as marked in Figure 5. An additional feature that has not been observed in the potential-controlled experiment (compare Figure 2) is the sample contraction upon hydrogen absorption in the galvanostatic experiment (black in Figure 5, enlarged in the inset). Figure 5 suggests that the presence of the $\mathrm{PdH}_{\beta}$-phase might have a critical influence on the irreversible length changes in npPd, which will be analysed in the Discussion section.

In order to evaluate structural coarsening, which is associated with a reduction in active surface area, cyclic voltammograms in the electrochemical double layer regime were recorded on an equivalent npPd sample before and after the thirteen-step absorption procedure, shown in Figure 5. Results are depicted in Figure 6. The CV after cyclic absorption (green curve) shows a reduction in double layer current of about $25 \%$ compared to the pristine, untreated npPd sample (black curve). The black curve shows a stronger contribution of pseudocapacitive surface charging, indicated by the larger deviation from the rectan-

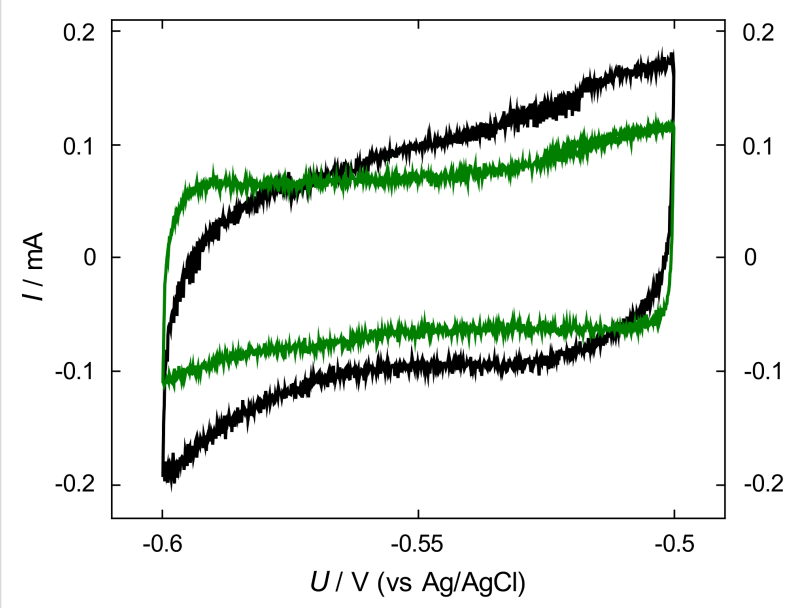

Figure 6: Current $I$ as a function of the applied potential $U$ during a single voltammetric cycle for nanoporous palladium in the electrochemical double-layer regime prior (black) and after cyclic hydrogen absorption following the charging sequence in Figure 5 (green). Voltammograms were recorded at a scan rate of $0.5 \mathrm{mV} \cdot \mathrm{s}^{-1}$ in $1 \mathrm{M} \mathrm{KOH}$. gular shape of ideal capacitors. A comparison of double-layer currents allows one to evaluate changes in the specific surface area of npPd upon hydrogenation (see section Discussion).

\section{Galvanostatic hydrogen desorption}

To investigate a possible dependence of the strain on the unloading rate, galvanostatic hydrogen-desorption experiments were performed, which are shown in Figure 7. For this purpose, a npPd sample was charged with hydrogen to a fixed concentration in the $\mathrm{PdH}_{\beta}$-phase and discharged at a fixed current, which in a first approximation should be proportional to the strain rate upon discharging. To achieve a defined loading state, a constant-current (green)/constant-potential (grey bars) charging procedure, inspired by a typical battery charging process, was implemented. As soon as the potential monitored during the constant-current ( $2 \mathrm{~mA}$ ) loading crossed $-1 \mathrm{~V}$, the potential was held constant at this value until the current dropped below $0.5 \mathrm{~mA}$. For the discharging experiments three different currents of $32 \mathrm{~mA}, 64 \mathrm{~mA}$, and $128 \mathrm{~mA}$ were applied. After each unloading step, a wait time of 5 min without external potential was set to allow for sample equilibration and desorption of residual hydrogen.

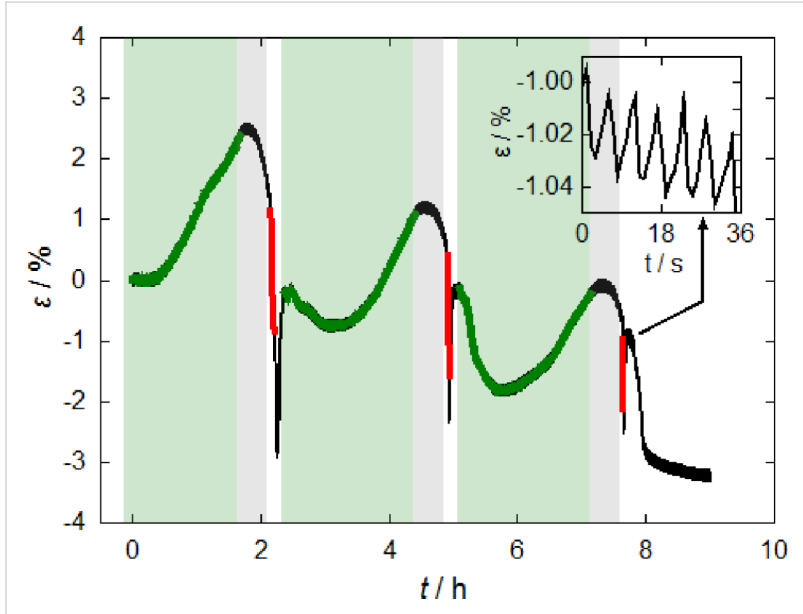

Figure 7: Strain $\varepsilon$ as a function of the time $t$ for constant-current/constant-potential ( $2 \mathrm{~mA}$ - green, $-1 \mathrm{~V}$ - grey bars) loading of hydrogen in nanoporous palladium followed by galvanostatic unloading at $32 \mathrm{~mA}$, $64 \mathrm{~mA}$ and $128 \mathrm{~mA}$ (red) in $1 \mathrm{M} \mathrm{KOH}$ solution. The inset shows an enlarged section of the curve after constant current desorption at $128 \mathrm{~mA}$.

The strain in Figure 7 shows a pronounced increase upon constant-current loading (green) followed by a small decrease during the constant-potential loading procedure (grey bars). Upon galvanostatic unloading (red) the strain drops almost linearly. Evaluation shows that the slope of these lines is approximately proportional to the unloading current. The black part of the curve directly after discharging, corresponds to the wait time, allowing the sample to relax. Once the strain passed 
the overshoot and reached a stable value, serrations (as shown in the inset) could be observed in the strain curve. These fluctuations were significantly larger than thermal fluctuations in the strain curves.

\section{Discussion}

In the following, the particular strain responses of npPd upon electrochemical hydrogen desorption, namely the strain overshoot, the strain offset and the high-current strain serrations, will be illuminated. The initial part of the discussion addresses the palladium-hydride phase transition, as the concept is of utmost importance for the mechanical behaviour of npPd.

\section{Current understanding of palladium-hydride phase transitions}

The nature of the phase transition in palladium hydride crucially depends on both sample dimensions and the rate of hydrogen uptake and removal. During hydrogen loading the $\mathrm{PdH}_{\alpha}$-to$\mathrm{PdH}_{\beta}$ transition was reported to proceed coherently in palladium nanoparticles, while the reverse transition can be either coherent or incoherent, depending on the particle size [34,35]. In bulk palladium the transition from $\mathrm{PdH}_{\beta}$ to $\mathrm{PdH}_{\alpha}$ is known to proceed incoherently, inducing dislocations to reduce internal stresses [34]. A coherent phase transition on the other hand involves the occurrence of internal stresses due to spatial variations of the lattice spacing, while no dislocations are induced. Therefore, particles must be sufficiently small in order to maintain surface-shell-core coherency during the $\alpha$-to- $\beta$ transition [34]. A study on phase transitions in $\mathrm{PdH}$ thin films concluded that coherent transitions are possible up to a critical film thickness of $22 \mathrm{~nm}$ [36]. As ligament sizes in npPd produced by dealloying in our work are in the order of $20 \mathrm{~nm}$, palladium hydride phase transitions can be expected to proceed mainly coherently as in nanoparticles up to a size of $45 \mathrm{~nm}$ [34], although ligament interconnections in the nanoporous network could enlarge the local structure size.

At low sorption rates it has been shown that upon hydrogen desorption the $\mathrm{PdH}_{\beta}$-to- $\mathrm{PdH}_{\alpha}$ transition follows a classical nucleation-and-growth mechanism, i.e., the $\alpha$-phase forms nuclei in the $\beta$-matrix that begin to grow [37]. During the hydride phase transition lattice constants of both phases may differ from their equilibrium values, which are quoted in the introduction. In situ $\mathrm{X}$-ray diffraction experiments pointed towards a reduced lattice constant for $\mathrm{PdH}_{\alpha}$ [38] when it appeared again during desorption. In the course of the desorption-induced phase transition the lattice constant of the $\alpha$-phase increases with the growing amount of $\mathrm{PdH}_{\alpha}$ (lattice-constant relaxation). When the $\mathrm{PdH}_{\beta}$ phase vanished, the lattice constant of $\mathrm{PdH}_{\alpha}$ was reported to be higher than its value before initial absorption, indicating an irreversible effect of the hydrogen treatment on the lattice [38]. It is important to note that also during absorption a decrease in lattice constant for $\mathrm{PdH}_{\alpha}$ was observed as soon as $\mathrm{PdH}_{\beta}$ started to form. The reduced lattice constant of the $\alpha$-phase, which will be referred to as $\alpha$-phase "straining" in the following, is related to internal stresses arising during the phase transition [37].

The driving force for the reduction of the lattice constant remains to be clarified, but an elastic compression of the $\alpha$-nuclei due to a structure-induced compressive stress at the solid-electrolyte interface is a plausible mechanism. As the $\mathrm{PdH}_{\alpha}$-phase nucleates in a $\mathrm{PdH}_{\beta}$-matrix, an additional compressive stress might be present due to the expanded lattice of the $\beta$-matrix, as already suggested in [38]. Both stresses, i.e., from the structure and from the $\beta$-matrix, could confine the $\alpha$-phase elastically to a smaller lattice constant.

When precipitates grow in the course of hydrogen desorption at a certain critical size a transition from coherent-to-incoherent (or coherent-to-semicoherent) is energetically favoured, which has been studied experimentally via small-angle neutron scattering [39]. The transition is expected not only to relief the stresses at the coherent $\alpha-\beta$ interfaces, but also the internal compressive stresses found in the $\alpha$-phase, leading to an increase in the lattice parameter of the $\alpha$-phase.

\section{a-phase straining - strain overshoot}

The strain overshoot visible in Figure 3, Figure 5 and Figure 7 can be explained by the aforementioned straining of the $\alpha$-phase (see previous section) as discussed in the following. The overshooting effect is most prominent during the desorption process in Figure 3. The monotonous length decrease in region (c) in Figure 3 can be attributed to hydrogen desorption from the $\mathrm{PdH}_{\beta}$-phase and a concomitant formation of strained $\mathrm{PdH}_{\alpha^{-}}$ nuclei. Region (d), where the strain starts to increase again, can be assigned to the process of lattice-constant relaxation in the $\alpha$-phase, while the fraction of $\mathrm{PdH}_{\beta}$ is reduced. Region (e) corresponds to the desorption from $\mathrm{PdH}_{\alpha}$, which is the predominant phase at this point, until the sample is completely dehydrogenated.

The same strain overshoot was monitored during desorption after galvanostatic loading and can be followed evolving over time in Figure 5. Considering a single absorption/desorption experiment as in the inset in Figure 5 one can easily recognise regions (c), (d) and (e) described above for Figure 3 in the red part.

In contrast to Figure 3, the straining of the $\alpha$-phase can be observed directly in the experiments upon absorption (black curves, Figure 5): Between cycles 5 and 11 the strain curves in black show a falling tendency after an initial increase. As the 
straining of the $\alpha$-phase is connected with internal stresses, it can only be observed in the region of phase coexistence of $\mathrm{PdH}_{\alpha}$ and $\mathrm{PdH}_{\beta}$. As soon as the voltage of $\mathrm{PdH}_{\alpha}$ depletion $(-0.99 \mathrm{~V})$ is crossed in the 11 th cycle of the galvanostatic loading series, a continuous length increase upon absorption due to expansion of the $\beta$-phase is observed in Figure 5, as a straining of $\mathrm{PdH}_{\alpha}$ is obviously no longer a possible mechanism.

In Figure 7 the strain overshoot can be monitored in a different form. Straining of the $\alpha$-phase occurs mainly in the region of constant-potential loading (grey bars in Figure 7), observable as decrease in the strain curve. Once the galvanostatic desorption starts (red) the sample contracts as expected with a strain rate approximately proportional to the unloading current. The relaxation of the strained $\alpha$-phase appears without an externally applied potential or current (black after unloading in red), resulting in a similar overshooting effect.

As indicated above, a possible straining of the $\mathrm{PdH}_{\alpha}$-regions in our experiments might be connected with the high surface stress exerted by the nanoporous structure, which could also account for the drastic changes in length even below the equilibrium value at the start of absorption (e.g., inset Figure 5, black curve). In this context it is worth mentioning a recent study on hierarchical npPd [20], which pointed towards a positive (tensile) surface stress in the $\mathrm{PdH}_{\beta}$-phase, thus a bulk contraction, and a negative (compressive) surface stress in the $\mathrm{PdH}_{\alpha^{-}}$ phase, thus a bulk expansion. This is in agreement with the straining of the $\mathrm{PdH}_{\alpha}$-phase in the $\mathrm{PdH}_{\beta}$-matrix, as a bulk compression by the $\mathrm{PdH}_{\beta}$-phase can also affect the $\mathrm{PdH}_{\alpha^{-}}$ nuclei, provided they have the lower volume fraction. Nucleation of the $\mathrm{PdH}_{\alpha}$ on the sample surface attenuates the compressive effect by $\mathrm{PdH}_{\beta}$, compared to nuclei surrounded by the $\beta$-phase. Overshooting behaviour is still plausible in this case, although with a reduced strain amplitude. Lattice reorientations driven by surface stress, suggested by simulations in both pris- tine [40] and hydrided Pd nanowires [41], could also be indicative for the concept of a surface-stress contribution to the $\alpha$-phase straining.

Finally, it should be noted that the stresses responsible for the overshooting behaviour must be different in nature from misfit stresses at a coherent phase boundary. As a $\alpha$-phase coherently matched to the $\beta$-phase would experience a tensile straining of the lattice constant, the above described compressive straining cannot be a result of these coherent interfaces.

\section{Internal-stress plasticity - strain offset}

The irreversible contractions after each absorption/desorption cycle observed in Figure $3(-0.5 \%)$ and Figure 5 (e.g., cycle 13, $-6.5 \%)$ are a result of the internal-stress plasticity mechanism that is elaborated below. The most important factor related to the irreversible contraction upon desorption after hydrogenation at low potentials are the forces which the sample is subjected to during dilatometry. On the one hand the sample experiences a weak, but steady compressive stress by means of the dilatometer pushrod. On the other hand, an additional driving force for compressive deformation due to the nanoporous structure itself and its surface excess energy is present in the sample. An effect called internal-stress plasticity (ISP), also commonly known as transformation plasticity, is well established in the literature [42-45]. A schematic representation of the transformation-mismatch plasticity mechanism in $\mathrm{npPd}$ is presented in Figure 8. A driving force for deformation, which is present from the two different sources in our case, biases internal strains emerging in the course of a phase transition in a preferred (radial) direction. This leads to an effective yielding in that direction [42]. We suppose that the transition from coherent to incoherent (or semicoherent) precipitates, triggers the ISP mechanism in our case. Both internal strains and a driving force for compression are necessary to activate this mechanism of plastic deformation.

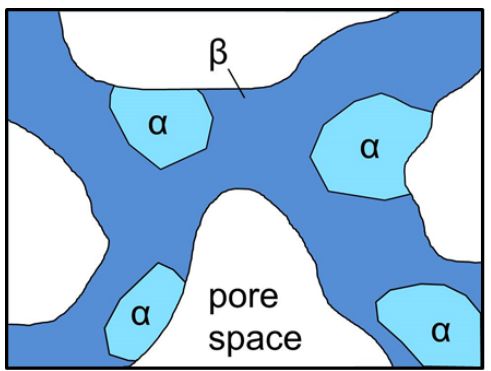

(1)

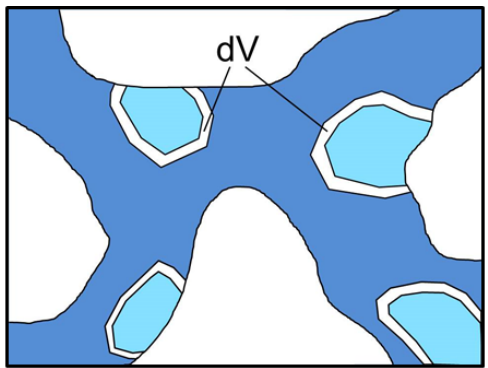

(2)

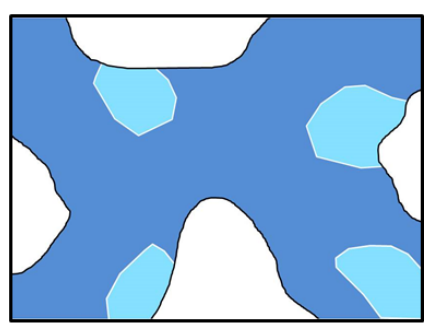

(3)

Figure 8: Schematic representation of the plasticity evolution during hydrogen desorption from nanoporous palladium hydride. In a saturated $\mathrm{PdH}_{\beta}$ phase $\mathrm{PdH}_{\alpha}$-nuclei start to form (1) in the course of the desorption process. The strain mismatch (dV) of the two phases emerging during the phase transition (2) is then biased by the surface tension and leads to plastic deformation of the nanoporous structure (3) in radial direction, so that surface area is reduced. Note that the total palladium volume remains unchanged during this transformation. 
The phase transition that induces the required mismatch strains for the plasticity enhancement is again the $\mathrm{PdH}_{\beta}$-to- $\mathrm{PdH}_{\alpha}$ transition. In combination with the driving force being the reduction in surface energy, ISP can be observed during hydrogen desorption in nanoporous samples. The high plastic-deformation strains up to $6.5 \%$ during one single phase-transition cycle in Figure 5 are a result of this mechanism. As the plastic deformation during a single absorption/desorption cycle is, in a firstorder approximation, proportional to the external stress [42,45], the strong deformation can be related to the surface-stress-induced compression in nanoporous samples, which is considerably higher than that in bulk samples. Hydrogen-induced phase transitions leading to ISP were reported for titanium [44], zirconium [46] and palladium [42] before. For a palladium wire an extraordinarily large deformation of $\approx 40 \%$ was reached under tensile load upon repetitive hydrogen loading and unloading in a long-term experiment [42]. In principle, such deformations should also be attainable for npPd by enlarging the number of absorption/desorption cycles.

Recent works on plasticity in nanoporous gold have pointed towards the surface tension (in units of energy per area) being the relevant capillary parameter for plastic deformation, rather than surface stress, which is only responsible for elastic contributions $[47,48]$. This is reflected in an asymmetric deformation behaviour in compression and tension as shown by Lührs et al. [48], where expansion under a tensile load is inhibited and a contraction under compressive force is promoted. Mameka et al. reported that surface-tension-driven deformation leads to changes in the total surface area, as supported by lower surface areas measured after compression tests [47]. Therefore, in our case of nanoporous palladium under compression a coarsening of the structure can be expected, giving rise to the length contraction. It has been shown using molecular dynamics (MD) simulations that plastic deformation at sufficiently high strains in general does promote structural coarsening in nanoporous materials [49]. The predicted coarsening of npPd upon (hydrogen-induced) deformation in our case is supported by CV measurements in the double-layer region (Figure 6). Double-layer currents from cyclic voltammograms are commonly used to calculate the electrochemical double-layer capacitance $C_{\mathrm{d}}$ and the related real surface area $A$, following the relation:

$$
A=\frac{C_{\mathrm{d}}}{C_{\mathrm{s}}}=\frac{I_{\mathrm{d}}}{s C_{\mathrm{s}}},
$$

where $C_{\mathrm{s}}$ denotes the specific capacitance per unit area, $I_{\mathrm{d}}$ the double-layer current and $s$ the scan rate at which the $\mathrm{CV}$ was recorded. As the capacitance per unit area $C_{\mathrm{s}}$ is a constant value for an electrode surface in a certain electrolyte, this capacitance values are directly proportional to real surface areas [50]. To date, no such reference values exist for palladium in potassium hydroxide solution. Nonetheless, the observed reduction of the double-layer current by about $25 \%$ in Figure 6 must lead to a surface area reduction of the same percentage. As the ligament diameter is related to the reciprocal specific suface area (SSA) in disorderd nanoporous structures [51], a decrease of SSA would give rise to an increase in ligament size. Assuming a constant relative density, one can give an upper boundary for the ligament-size increase of about 33\% [51]. The initial ligament size of approximately $20 \mathrm{~nm}$ should accordingly coarsen to ligament sizes not larger than $27 \mathrm{~nm}$. This increase in ligament size, derived from double layer current variations, is a strong indication that the ISP deformation mechanism is active in $\mathrm{npPd}$.

Plastic deformation up to $6.5 \%$ after a single hydrogen-loading/ unloading cycle in nanoporous palladium samples prepared in this work (see Figure 5) is seemingly in striking contrast with the remarkable hydrogen-cycling stability over 1000 cycles reported for hierarchical nanoporous palladium by Shi et al. [20]. However, closer inspection suggests that the mechanism proposed here leading to plastic deformation might not be available in hierarchical npPd prepared in the mentioned work. The smaller structural size of the finer ligament structure below $10 \mathrm{~nm}$ in the work of Shi et al. might allow for a fully coherent phase transition completely avoiding the creation of incoherent phase boundaries and thus defects also upon desorption, which is an idea already brought up by the authors of the mentioned publication. In the case of npPd produced in this work phase coherency is only partly possible upon hydrogen desorption for our nanoporous samples indicated, e.g., by serrations in the strain (see next section). As the transition from coherent-toincoherent (or coherent-to-semicoherent) interfaces of $\mathrm{PdH}_{\alpha}$ and $\mathrm{PdH}_{\alpha}$ phases is supposed to initiate deformation, this could explain the discrepancies between the mechanical performance of the two variants of npPd. In addition the contribution of dislocation plasticity is avoided when the phase transition proceeds fully coherently.

Crossing the threshold potential of $\mathrm{PdH}_{\beta}$-phase formation $(-0.96 \mathrm{~V})$ in our electrochemical experiments enables plastic deformation following the ISP mechanism, which is the foundation of plasticity control in npPd. Only if the $\beta$-phase is formed upon hydrogenation (starting in cycle 5 in Figure 5) plasticity occurs during the desorption process. Theoretical aspects of the strain response, including both surface stress and plasticity mechanism, are discussed in the Appendix section.

\section{Dislocation plasticity - strain serrations}

The strain serrations during high-current desorption processes, visible in the inset in Figure 7, indicates the presence of an ad- 
ditional deformation mechanism of npPd based on dislocation activity, which is subject of this section.

Two different mechanisms of plasticity evolution are common in hydrogen-treated metals: hydrogen embrittlement $[52,53]$ and hydrogen-enhanced localised plasticity [54]. In a simplistic view, hydrogen embrittlement evolves as a result of dislocations introduced by interstitial hydrogen atoms, which remain in the crystal lattice and hinder dislocation movement, even after complete hydrogen desorption in an ideal lattice. Hydrogen-enhanced localised plasticity, on the other hand, is a result of stress-field shielding by solute hydrogen atoms and thus an enhancement of dislocation mobility. The appearance of these converse phenomena strongly depends on the grain size. In small grains hydrogen-enhanced localised plasticity is dominant, while in coarse grains hydrogen embrittlement is the prevalent mechanism [55]. Hydrogen-metal interactions are still not fully understood and demand further research.

The ISP mechanism, responsible for the irreversible contraction (strain offset, see previous section), is based on the coherency of phases during the transition, which does not introduce dislocations in the nanoporous structure. However, a fully coherent transition can be hampered at high sorption and desorption rates, which may give rise to additional dislocation plasticity.

The strong fluctuations observed in the inset in Figure 7 point towards a dislocation plasticity contribution. Serrations are common features in stress-strain curves as a result of dynamic strain aging (DSA) [56]. DSA is related to dislocation interactions with obstacles in the lattice, which may be other lattice defects or solute atoms. The effect is activated at high strain (and thus desorption) rates, where solutes fail to keep up with the rapidly moving dislocations [56]. Hydrogen solute atoms were reported to cause DSA in $\alpha$ - Ti [56] at low concentrations. The Portevin-Le Chatelier effect, which is directly related to the DSA mechanism, was also reported for hydrogenated palladium [57]. Arrested dislocations might also be possible in $\mathrm{npPd}$. Hydrogen is present as solute atom in the $\mathrm{PdH}_{\alpha}$-phase, which is the predominant phase after (incomplete) galvanostatic desorption. Arrested dislocation movement, however, cannot solely account for the sawtooth-shape of the strain curve in the inset in Figure 7. Since dislocation arrest simply corresponds to a constant sample length, the strain curve would decrease in a steplike manner, but no length increase would be observed as it is the case in Figure 7. Nonetheless, a combination of dislocation arrest, being responsible for the descending part of the sawtooth, with the $\mathrm{PdH}_{\alpha}$-phase relaxation introduced above, accounting for the ascending part of the sawtooth, could be a possible explanation for the serrations in the strain curve.

\section{Summary}

In this work we investigated the deformation mechanisms in npPd by using an in situ dilatometric technique in an electrochemical environment. Different hydrogen-sorption experiments, controlled by either current or potential, were used to induce phase transitions in npPd in a controlled manner and to evaluate the influence of $\mathrm{PdH}_{\alpha}$ - and $\mathrm{PdH}_{\beta}$-phase on the strain response. Plasticity mechanisms based on both internal stresses and dislocation activity were found to be active in nanoporous palladium hydrides, leading to a peculiar strain curve. A phasetransition upon dehydrating in combination with the extraordinarily high surface stress, due to the nanoporous structure, is responsible for this uncommon strain response. A maximum compressive plastic deformation of $\approx 6.5 \%$ could be attained during a single hydrogen sorption-desorption-cycle. A phenomenological description of plastic deformation in npPd led to the following conclusions:

- The driving force of plastic deformation in nanoporous palladium is the surface stress.

- A coherent hydrogen-induced phase transition from $\mathrm{PdH}_{\beta}$ to $\mathrm{PdH}_{\alpha}$ during hydrogen desorption enables the mechanism of internal-stress plasticity in nanoporous palladium.

- This phase transition, and thus plasticity, can be controlled via the potential in an electrochemical environment. A threshold potential for nanoporous $\mathrm{PdH}_{\beta}$ formation $(-0.96 \mathrm{~V}$ vs $\mathrm{Ag} / \mathrm{AgCl}$ in $1 \mathrm{M} \mathrm{KOH})$ was determined.

\section{Experimental}

\section{Alloy fabrication}

A $\mathrm{Co}_{75} \mathrm{Pd}_{25}$ master alloy was prepared from Pd granules (AlfaAesar, 99.95\%) and Co slug (AlfaAesar, 99.95\%) via electron-beam melting. The sample was melted multiple times to ensure complete intermixing in the liquid state and consequently a homogeneous single-phase alloy. The produced alloy drop was thinned to a platelet (4-5 $\mathrm{mm}$ in height) using a screw press. Homogeneity of the alloy was confirmed by XRD-measurements. Further processing consisted of several consecutive rolling and annealing steps until a thickness of $270 \mu \mathrm{m}$ was reached. The annealing steps were conducted in a vacuum furnace at $700{ }^{\circ} \mathrm{C}$ and $10^{-5}$ mbar for $1 \mathrm{~h}$. The resulting foil was cut into squares of $5 \times 5 \mathrm{~mm}^{2}$.

\section{Dealloying}

The setup used for in situ dilatometry during electrochemical characterisation was similar to that described in an earlier work of our group [17]. For electrochemical dealloying the squareshaped samples were placed in a Linseis L75 vertical pushrod dilatometer, operating at a constant force of $100 \mathrm{mN}$. Electrical 
contact to an Autolab PGSTAT204 potentiostat was established using an annealed Pd wire (ChemPur, 99.95\%). A coiled Pd wire and an $\mathrm{Ag} / \mathrm{AgCl}$ (sat. $\mathrm{KCl}$ ) electrode (Metrohm) served as counter and reference electrode, respectively. Electrochemical dealloying was conducted in $0.1 \mathrm{M}$ sulfuric acid solution at a potential of $+0.55 \mathrm{~V}$ ( $\mathrm{vs} \mathrm{Ag} / \mathrm{AgCl}$ ), a method that is commonly used to achieve homogeneous nanoporous palladium structures $[16,17]$. Dealloying was stopped at currents below $0.1 \mathrm{~mA}$. The residual cobalt concentration in nanoporous palladium prepared via this route was reported to be below 2 atom \% [58].

\section{Electrochemical cell setup}

After dealloying, the samples were rinsed in distilled water for several minutes, before immersing them in $1 \mathrm{M} \mathrm{KOH}$ aqueous solution. For electrochemical characterisation a porous carbon cloth was used as counter electrode. In order to assure measurement stability a pre-treatment consisting of five voltammetric cycles was applied at a scan rate of $0.1 \mathrm{mV} \cdot \mathrm{s}^{-1}$ in a potential window between $-1 \mathrm{~V}$ and $0.4 \mathrm{~V}$. All potentials in the text below refer to the $\mathrm{Ag} / \mathrm{AgCl}$ (sat. $\mathrm{KCl}$ ) reference electrode. Zero on the strain axis in the plots was chosen in the electrochemical double-layer regime. For the distinction of hydride phases in $n p P d$, samples were dealloyed and characterised ex situ in a standard three-electrode electrochemical cell, using the same counter and reference electrodes as described for the in situ setup.

As chemical dissolution processes can hardly be discerned from mechanical yielding on the basis of strain curves obtained in a dilatometer, dissolution processes should be minimised using an adequate electrolyte. Aqueous potassium hydroxide solution enables measurement stability in a broad potential window $[17,18]$, while mostly surpressing chemical dissolution of palladium and palladium oxide [29], making it the preferred electrolyte for studies in this work.

\section{Appendix}

\section{Theoretical considerations}

The theoretical basis to evaluate the experimental strain response of npPd upon hydrogen absorption and desorption will be summarised in the following. As indicated above, the irreversible, plastic length changes of nanoporous palladium measured in the dilatometer are results of two different stresses: the stress resulting from the external force applied by the dilatometer $\left(\sigma_{\mathrm{dil}}\right)$ and the axial component of the surface stress of the nanoporous structure $\left(\sigma_{\mathrm{ss}}\right) . \sigma_{\mathrm{dil}}$ is of the order of $10 \mathrm{kPa}$ for dilatometric experiments, while $\sigma_{\mathrm{ss}}$ is related to the ligament size reciprocally:

$$
\sigma_{\mathrm{SS}}=\frac{3.7 f}{D} .
$$

The factor 3.7 is a dimensionless constant calculated for arbitrary porous structures [51], $f$ is the surface stress (for a Pd surface: $f \approx 1.9 \mathrm{~N} / \mathrm{m}[59]$ ) and $D$ the ligament size. With ligament sizes of the order of $20 \mathrm{~nm}$, an axial component of the surface stress of about $350 \mathrm{MPa}$ would be obtained. Considering this high value one can exclude a significant contribution of the dilatometric stress to the deformation in nanoporous palladium. Both stresses contribute to elastic and plastic deformation of the sample. An expression for the elastic component of the length changes in a nanoporous material, which has been introduced for nanoporous gold recently [60], is

$$
\Delta \varepsilon_{\mathrm{e}}=-\frac{2 \alpha}{9 K} \Delta f-\frac{\sigma_{\mathrm{dil}}}{E_{\mathrm{np}}}
$$

where $\Delta \varepsilon_{\mathrm{e}}$ denotes the change in elastic strain, $\alpha$ is the specific surface area $A / V, K$ the bulk modulus, $\Delta f$ the change in surface stress, and $E_{\mathrm{np}}$ the Young's modulus of the nanoporous structure. $\Delta f$ can be linked to the change in surface charge density $\Delta q$ via electrocapillary coupling coefficients $\zeta$ in different electrochemical regimes. Similar relationships have been successfully utilised to bias dilatometric elastic-strain responses by means of electrochemical surface charging in nanoporous systems $[3,61$ 63].

Note that the absolute value of the surface stress $(f)$ does not contribute to any elastic length changes, but variations of this quantity do $(\Delta f)$. The plastic component of the total strain, which is a result of the ISP mechanism, is described by the theoretical model of Greenwood and Johnson [45] for sufficiently low stresses compared to the yield strength. In the present work we propose a slightly altered version of this relationship, linking plastic deformation to surface stress:

$$
\Delta \varepsilon_{\mathrm{p}}=\frac{5}{3} \frac{\Delta V}{V} \frac{\left(\sigma_{\mathrm{dil}}+\sigma_{\mathrm{ss}}\right)}{\sigma_{\mathrm{y}}}
$$

where $\Delta \varepsilon_{\mathrm{p}}$ is the plastic strain per phase-transformation cycle, $\Delta V / V$ the volume mismatch of the two involved phases (for Pd and $\mathrm{PdH}_{\beta}: \Delta V / V \approx 0.1$ ), and $\sigma_{\mathrm{y}}$ the yield strength of the nanoporous palladium. As pointed out in the discussion the actual driving force for plastic deformation is surface tension. From surface tension, which is a scalar quantity, it is not possible to determine a corresponding surface stress, which is a tensor quantity, without additional information. The surface stress $\sigma_{\mathrm{ss}}$ in Equation 5 therefore refers to a virtual stress, representing the effect of surface tension on plastic deformation. 
The yield strength of npPd also strongly depends on the ligament size, following a relation similar to the Hall-Petch equation [64]:

$$
\sigma_{\mathrm{y}}=\sigma_{0}+k L^{-0.5}
$$

$\sigma_{0}$ denotes the yield strength of bulk palladium and $k$ is a material-specific constant, yet to be determined for nanoporous palladium. It has been shown for $\mathrm{npAu}$, which follows a similar scaling equation for both yield strength and surface stress, that $\sigma_{\mathrm{SS}}$ is well below $\sigma_{\mathrm{y}}$ for ligament sizes down to $10 \mathrm{~nm}$ [24] This allows to use Equation 5, which is only valid in the regime of low stresses. At larger stresses the plastic strain is connected with the applied stress via a more complex, nonlinear relationship [43]. The plastic strain component is, in contrast to the elastic deformation, proportional to the absolute value of the surface stress $(f)$ in our proposed equation (Equation 5). Adding a term accounting for the work-hardening process during a phase transition should be considered for a more accurate description of the property evolution.

Many properties in the above-mentioned equations can not be assumed to be constant during our experiments. Besides the potential-dependence of the surface stress $f$, which is utilised to trigger the strain response, it is well known that $E_{\mathrm{np}}, D, \alpha$ and $\sigma_{\mathrm{np}}$ are all potential-dependent properties, which makes the above-stated equations hard to evaluate during measurements in practice. The given framework of equations might serve as a starting point for a detailed theoretical treatment of the mechanical properties of npPd in the future.

\section{Acknowledgements}

This work was financially supported by the Austrian Science Fund (FWF): P30070-N36.

\section{ORCID ${ }^{\circledR}$ iDs}

Markus Gößler - https://orcid.org/0000-0003-3731-3118 Eva-Maria Steyskal - https://orcid.org/0000-0003-0752-9997 Markus Stütz - https://orcid.org/0000-0001-9400-5168 Norbert Enzinger - https://orcid.org/0000-0003-0051-9518 Roland Würschum - https://orcid.org/0000-0003-4624-4433

\section{References}

1. Biener, J.; Wittstock, A.; Zepeda-Ruiz, L. A.; Biener, M. M.; Zielasek, V.; Kramer, D.; Viswanath, R. N.; Weissmüller, J.; Bäumer, M.; Hamza, A. V. Nat. Mater. 2009, 8, 47-51. doi:10.1038/nmat2335

2. Jin, H.-J.; Weissmüller, J. Adv. Eng. Mater. 2010, 12, 714-723. doi:10.1002/adem.200900329

3. Jin, H.-J.; Parida, S.; Kramer, D.; Weissmüller, J. Surf. Sci. 2008, 602, 3588-3594. doi:10.1016/j.susc.2008.09.038
4. Steyskal, E.-M.; Besenhard, M.; Landgraf, S.; Zhong, Y.; Weissmüller, J.; Pölt, P.; Albu, M.; Würschum, R. J. Appl. Phys. 2012, 112, 073703. doi:10.1063/1.4755808

5. Steyskal, E.-M.; Topolovec, S.; Landgraf, S.; Krenn, H.; Würschum, R. Beilstein J. Nanotechnol. 2013, 4, 394-399. doi:10.3762/bjnano.4.46

6. Steyskal, E.-M.; Qi, Z.; Pölt, P.; Albu, M.; Weissmüller, J.; Würschum, R. Langmuir 2016, 32, 7757-7764. doi:10.1021/acs.langmuir.6b01734

7. Ghosh, S.; Lemier, C.; Weissmüller, J. IEEE Trans. Magn. 2006, 42, 3617-3619. doi:10.1109/tmag.2006.880922

8. Jalas, D.; Shao, L.-H.; Canchi, R.; Okuma, T.; Lang, S.; Petrov, A.; Weissmüller, J.; Eich, M. Sci. Rep. 2017, 7, 44139. doi:10.1038/srep44139

9. McCurry, D. A.; Bailey, R. C. J. Phys. Chem. C 2016, 120, 20929-20935. doi:10.1021/acs.jpcc.6b02759

10. Erlebacher, J.; Aziz, M. J.; Karma, A.; Dimitrov, N.; Sieradzki, K. Nature 2001, 410, 450-453. doi:10.1038/35068529

11. Liu, Y.; Bliznakov, S.; Dimitrov, N. J. Electrochem. Soc. 2010, 157, K168-K176. doi:10.1149/1.3454753

12. Parida, S.; Kramer, D.; Volkert, C. A.; Rösner, H.; Erlebacher, J.; Weissmüller, J. Phys. Rev. Lett. 2006, 97, 035504. doi:10.1103/physrevlett.97.035504

13. Steyskal, E.-M.; Seidl, M.; Graf, M.; Würschum, R. Phys. Chem. Chem. Phys. 2017, 19, 29880-29885. doi:10.1039/c7cp05706g

14. Senior, N. A.; Newman, R. C. Nanotechnology 2006, 17, 2311-2316. doi:10.1088/0957-4484/17/9/040

15. McCue, I.; Benn, E.; Gaskey, B.; Erlebacher, J. Annu. Rev. Mater. Res. 2016, 46, 263-286. doi:10.1146/annurev-matsci-070115-031739

16. Hakamada, M.; Mabuchi, M. J. Alloys Compd. 2009, 479, 326-329. doi:10.1016/j.jallcom.2008.12.078

17. Steyskal, E.-M.; Wiednig, C.; Enzinger, N.; Würschum, R. Beilstein J. Nanotechnol. 2016, 7, 1197-1201. doi:10.3762/bjnano.7.110

18. Zhang, J.; Wang, Y.; Si, C.; Bai, Q.; Ma, W.; Gao, H.; Zhang, Z. Electrochim. Acta 2016, 220, 91-97. doi:10.1016/j.electacta.2016.10.091

19. Zhang, J.; Bai, Q.; Zhang, Z. Nanoscale 2016, 8, 7287-7295. doi:10.1039/c6nr00427j

20. Shi, S.; Markmann, J.; Weissmüller, J. Philos. Mag. 2017, 97 , 1571-1587. doi:10.1080/14786435.2017.1311428

21. Manchester, F. D.; San-Martin, A.; Pitre, J. M. J. Phase Equilib. 1994, 15, 62-83. doi:10.1007/bf02667685

22. Wagner, S.; Pundt, A. Int. J. Hydrogen Energy 2016, 41, 2727-2738. doi:10.1016/j.ijhydene.2015.11.063

23. Ramanathan, M.; Skudlarek, G.; Wang, H. H.; Darling, S. B. Nanotechnology 2010, 21, 125501. doi:10.1088/0957-4484/21/12/125501

24. Ye, X.-L.; Jin, H.-J. Appl. Phys. Lett. 2013, 103, 201912. doi:10.1063/1.4831686

25. Sun, S.; Chen, X.; Badwe, N.; Sieradzki, K. Nat. Mater. 2015, 14, 894-898. doi:10.1038/nmat4335

26. Jin, H.-J.; Weissmüller, J. Science 2011, 332, 1179-1182. doi:10.1126/science.1202190

27. Greer, J. R.; Nix, W. D. Phys. Rev. B 2006, 73, 245410. doi:10.1103/physrevb.73.245410

28. Ngô, B.-N. D.; Stukowski, A.; Mameka, N.; Markmann, J.; Albe, K.; Weissmüller, J. Acta Mater. 2015, 93, 144-155. doi:10.1016/j.actamat.2015.04.021 
29. Grdeń, M.; Łukaszewski, M.; Jerkiewicz, G.; Czerwiński, A. Electrochim. Acta 2008, 53, 7583-7598.

doi:10.1016/j.electacta.2008.05.046

30. Singh, R. K.; Ramesh, R.; Devivaraprasad, R.; Chakraborty, A.; Neergat, M. Electrochim. Acta 2016, 194, 199-210. doi:10.1016/j.electacta.2016.01.231

31. Łukaszewski, M.; Grdeń, M.; Czerwiński, A. J. New Mater. Electrochem. Syst. 2006, 9, 409-417.

32. Łukaszewski, M.; Klimek, K.; Żurowski, A.; Kędra, T.; Czerwiński, A. Solid State Ionics 2011, 190, 18-24. doi:10.1016/j.ssi.2010.03.009

33. Eastman, J. A.; Thompson, L. J.; Kestel, B. J. Phys. Rev. B 1993, 48, 84-92. doi:10.1103/physrevb.48.84

34. Griessen, R.; Strohfeldt, N.; Giessen, H. Nat. Mater. 2016, 15, 311-317. doi:10.1038/nmat4480

35. Narayan, T. C.; Hayee, F.; Baldi, A.; Leen Koh, A.; Sinclair, R.; Dionne, J. A. Nat. Commun. 2017, 8, 14020. doi: $10.1038 /$ ncomms 14020

36. Wagner, S.; Uchida, H.; Burlaka, V.; Vlach, M.; Vlcek, M.; Lukac, F.; Cizek, J.; Baehtz, C.; Bell, A.; Pundt, A. Scr. Mater. 2011, 64, 978-981. doi:10.1016/j.scriptamat.2011.02.004

37. Goltsova, M. V.; Artemenko, Yu. A.; Zhirov, G. I.; Zaitsev, V. I. Int. J. Hydrogen Energy 2002, 27, 757-763 doi:10.1016/s0360-3199(01)00104-5

38. Kawasaki, A.; Itoh, S.; Shima, K.; Kato, K.; Ohashi, H.; Ishikawa, T.; Yamazaki, T. Phys. Chem. Chem. Phys. 2015, 17, 24783-24790. doi:10.1039/c5cp02725j

39. Chen, W. C.; Heuser, B. J.; King, J. S. J. Appl. Crystallogr. 2000, 33, 442-446. doi:10.1107/s0021889899013667

40. Lao, J.; Moldovan, D. Appl. Phys. Lett. 2008, 93, 093108. doi:10.1063/1.2976434

41. He, J. H.; Knies, D. L.; Hubler, G. K.; Grabowski, K. S.; Tonucci, R. J.; Dechiaro, L. F. Appl. Phys. Lett. 2012, 101, 153103. doi:10.1063/1.4757999

42. Beeri, O.; Dunand, D. C. Mater. Sci. Eng., A 2009, 523, 178-183. doi:10.1016/j.msea.2009.05.048

43. Zwigl, P.; Dunand, D. C. Acta Mater. 1997, 45, 5285-5294. doi:10.1016/s1359-6454(97)00186-9

44. Dunand, D. C.; Zwigl, P. Metall. Mater. Trans. A 2001, 32, 841-847. doi:10.1007/s11661-001-1019-4

45. Greenwood, G. W.; Johnson, R. H. Proc. R. Soc. London, Ser. A 1965, 283, 403-422. doi:10.1098/rspa.1965.0029

46. Hong, E.; Dunand, D. C.; Choe, H. Int. J. Hydrogen Energy 2010, 35 , 5708-5713. doi:10.1016/j.ijhydene.2010.03.088

47. Mameka, N.; Markmann, J.; Weissmüller, J. Nat. Commun. 2017, 8, 1976. doi:10.1038/s41467-017-01434-2

48. Lührs, L.; Zandersons, B.; Huber, N.; Weissmüller, J. Nano Lett. 2017, 17, 6258-6266. doi:10.1021/acs.nanolett.7b02950

49. Kolluri, K.; Demkowicz, M. J. Acta Mater. 2011, 59, 7645-7653. doi:10.1016/j.actamat.2011.08.037

50. Fang, L.-I.; Tao, Q.; Li, M.-f.; Liao, L.-w.; Chen, D.; Chen, Y.-x. Chin. J. Chem. Phys. 2010, 23, 543-548. doi:10.1088/1674-0068/23/05/543-548

51. Detsi, E.; De Jong, E.; Zinchenko, A.; Vuković, Z.; Vuković, I.; Punzhin, S.; Loos, K.; ten Brinke, G.; De Raedt, H. A.; Onck, P. R.; De Hosson, J. T. M. Acta Mater. 2011, 59, 7488-7497. doi:10.1016/j.actamat.2011.08.025

52. Dillon, E.; Jimenez, G.; Davie, A.; Bulak, J.; Nesbit, S.; Craft, A. Mater. Sci. Eng., A 2009, 524, 89-97. doi:10.1016/j.msea.2009.07.036
53. Carpena-Núñez, J.; Yang, D.; Kim, J.-W.; Park, C.; Fonseca, L. F. Nanotechnology 2013, 24, 035701. doi:10.1088/0957-4484/24/3/035701

54. Sofronis, P.; Birnbaum, H. Hydrogen enhanced localized plasticity: A mechanism for hydrogen related fracture. In Fatigue and Fracture of Aerospace Structural Materials; Nagar, A.; Kuo, A.-Y., Eds.; ASME, 1993; pp 15-25.

55. Hongo, T.; Edalati, K.; Iwaoka, H.; Arita, M.; Matsuda, J.; Akiba, E.; Horita, Z. Mater. Sci. Eng., A 2014, 618, 1-8. doi:10.1016/j.msea.2014.08.074

56. Senkov, O. N.; Jonas, J. J. Metall. Mater. Trans. A 1996, 27, 1877-1887. doi:10.1007/bf02651937

57. Blakemore, J. S.; Hall, E. O. J. Nucl. Mater. 1970, 34, 114-115. doi:10.1016/0022-3115(70)90017-6

58. Hakamada, M.; Nakano, H.; Furukawa, T.; Takahashi, M.; Mabuchi, M. J. Phys. Chem. C 2010, 114, 868-873. doi:10.1021/jp909479m

59. Skriver, H. L.; Rosengaard, N. M. Phys. Rev. B 1992, 46, 7157-7168. doi:10.1103/physrevb.46.7157

60. Liu, F.; Ye, X.-L.; Jin, H.-J. Phys. Chem. Chem. Phys. 2017, 19, 19217-19224. doi:10.1039/c7cp03033a

61. Viswanath, R. N.; Kramer, D.; Weissmüller, J. Electrochim. Acta 2008, 53, 2757-2767. doi:10.1016/j.electacta.2007.10.049

62. Viswanath, R. N.; Weissmüller, J. Acta Mater. 2013, 61, 6301-6309. doi:10.1016/j.actamat.2013.07.013

63. Weissmüller, J.; Viswanath, R. N.; Kramer, D.; Zimmer, P.; Würschum, R.; Gleiter, H. Science 2003, 300, 312-315. doi:10.1126/science. 1081024

64. Hodge, A. M.; Biener, J.; Hayes, J. R.; Bythrow, P. M.; Volkert, C. A.; Hamza, A. V. Acta Mater. 2007, 55, 1343-1349. doi:10.1016/j.actamat.2006.09.038

\section{License and Terms}

This is an Open Access article under the terms of the Creative Commons Attribution License (http://creativecommons.org/licenses/by/4.0). Please note that the reuse, redistribution and reproduction in particular requires that the authors and source are credited.

The license is subject to the Beilstein Journal of Nanotechnology terms and conditions: (https://www.beilstein-journals.org/bjnano)

The definitive version of this article is the electronic one which can be found at: doi:10.3762/bjnano.9.280 